\title{
РАСПРОСТРАНЕНИЕ ЗВУКА
}

\author{
В.Г. Бурдуковская, М.А. Раевский
}

\section{ВЛИЯНИЕ АНИЗОТРОПИИ ВЕТРОВОГО ВОЛНЕНИЯ НА ЭФФЕКТЫ РАССЕЯНИЯ АКУСТИЧЕСКИХ МОД В МЕЛКОМ МОРЕ}

\author{
Институт прикладной физики Российской академии наук \\ Россия, 603950, Нижний Новгород, ул. Ульянова, 46 \\ Тел.: (831) 416-0629; E-mail: bvg@appl.sci-nnov.ru
}

\begin{abstract}
Проведено теоретическое исследование горизонтальной анизотропии акустических характеристик тонального сигнала в волноводе с анизотропным ветровым волнением. Обсуждается применимость традиционной модели изотропного ветрового волнения для задач прикладной акустики.
\end{abstract}

Ключевые слова: акустические характеристики, изотропное ветровое волнение

Как правило, эффекты многократного рассеяния акустического поля на ветровом волнении исследуются с использованием упрощенной модели изотропного ветрового волнения. Вместе с тем хорошо известно, что реальное ветровое волнение имеет высокую степень анизотропии, что подтверждено многочисленными натурными экспериментами [3]. Ранее было проведено теоретическое исследование влияния анизотропии волнения на горизонтальный угловой спектр динамических шумов океана [4]. Здесь мы рассмотрим влияние анизотропии ветрового волнения на статистические характеристики акустических мод, возбуждаемых тональным источником. Основное внимание уделено сравнению результатов, прогнозируемых для модели ветрового анизотропного волнения с эмпирическим частотно-угловым спектром и упрощенной модели изотропного волнения. 
Рассмотрим акустическое поле, генерируемое тональным источником с частотой $f_{0}$ в океаническом волноводе, свободная граница которого имеет случайные вертикальные смещения, обусловленные ветровым волнением. Для акустики мелкого моря основной интерес представляет низкочастотный диапазон $f \leq 300$ Гц. При этом для типичных скоростей ветра $V<<15$ м/с среднеквадратичная амплитуда волнения и частота $\omega_{0}$, как правило, удовлетворяют условию малости параметра Рэлея. В случае модового описания поля это условие означает малость эффектов однократного рассеяния акустических мод, но позволяет описывать эффекты многократного рассеяния.

Исходным для анализа является уравнение переноса для функций поперечной корреляции нормальных мод в волноводе с нерегулярным профилем свободной границы. Для ненаправленного в горизонтальной плоскости источника оно имеет вид:

$$
\frac{\partial N_{p}(\rho, x)}{\partial x}=\sum_{p_{2}} W_{p p_{2}}(\rho, x) N_{p_{2}}(\rho, x)-2\left(\gamma_{p}+\operatorname{Im} k_{p}\right) N_{p}(\rho, x)(1)
$$

Здесь $\gamma_{p}$ - декремент затухания когерентной компоненты амплитуды моды $<a_{\mathrm{p}}>$, Im $k_{\mathrm{p}}-$ мнимая часть волнового числа, обусловленная потерями энергии в воде и донном грунте, $W_{\mathrm{pp2}}$ - вероятность перехода между модами, описывающая эффекты взаимного рассеяния мод, локализованных в волноводе, $N_{\mathrm{p}}(\rho, x)$ - функция корреляции амплитуд мод:

$$
N_{p}(\rho, x)=\left\langle a_{p}\left(-\frac{\rho}{2}, x\right) a_{p}^{*}\left(\frac{\rho}{2}, x\right)\right\rangle .
$$

Функции $\gamma_{p}$ и $W_{\mathrm{pp} 2}$ выражаются через частотно-угловой спектр ветрового волнения [4].

Частотно-угловой спектр волнения $B(\Omega, \theta)$, как правило, рассматривается в виде произведения частотного спектра $F(\Omega)$ и нормированного углового спектра $Q(\Omega, \theta)$ :

$$
B(\Omega, \theta)=F(\Omega) Q(\Omega, \theta)
$$

В настоящее время достаточно хорошо изучен эмпирический частотный спектр волнения $F(\Omega)$, для которого используется модель JONSWAP [3]. Для функции $Q(\Omega, \theta)$ обычно используют аппроксимацию:

$$
Q(\Omega, \theta)=2^{2 s-1} \frac{\Gamma^{2}(s+1)}{\Gamma(2 s+1)}\left[\cos \left(\frac{\theta-\alpha}{2}\right)\right]^{2 s} .
$$


Здесь $\alpha$ - направление ветра, $\Gamma(x)$ - гамма-функция, а $s$ зависит от частоты $\Omega$. Здесь мы будем использовать эмпирическую зависимость [3]:

$$
\begin{aligned}
& s=11.5\left(\frac{g}{V}\right)^{2.5} \Omega_{m}^{-7.5} \Omega^{5} \text { при } \Omega \leq \Omega_{m} ; \\
& s=11.5\left(\frac{g}{V}\right)^{2.5} \Omega^{-2.5} \text { при } \Omega>\Omega_{m}
\end{aligned}
$$

Уравнение (1) может быть решено лишь численными методами. Далее мы ограничимся рассмотрением когерентной компоненты модовых амплитуд и интенсивности рассеянной компоненты мод в мелком море. Численное моделирование было выполнено для гидрологических условий Баренцева моря в зимний период, для которого характерны профили скорости звука со слабым положительным градиентом. Расчеты проведены для волновода с линейным профилем скорости звука: $\mathrm{c}(0)=$ $1490 \mathrm{~m} / \mathrm{c}, \mathrm{c}(\mathrm{H})=1500 \mathrm{~m} / \mathrm{c}$., глубина дна $H=200$ м. В качестве моде-

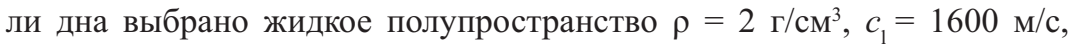
$c_{t}=0 \mathrm{~m} / \mathrm{c}$. Предполагается, что глубина точечного источника $z_{\mathrm{И}}=10 \mathrm{M}$, частота излучения $f_{0}=240$ Гц. Для скорости ветра V выбиралось типичное значение $V=10 \mathrm{~m} / \mathrm{c}$, что соответствует умеренному ветровому волнению.

Поскольку нас интересует отличие результатов, полученных для анизотропной модели ветрового волнения и упрощенной модели изотропного волнения, далее приводятся отношения соответствующих значений. На рис. 1 приведены нормированные таким образом значения декремента затухания когерентной компоненты $\gamma_{p}(\alpha)$ для мод с номерами $p=1,10$ и 20.

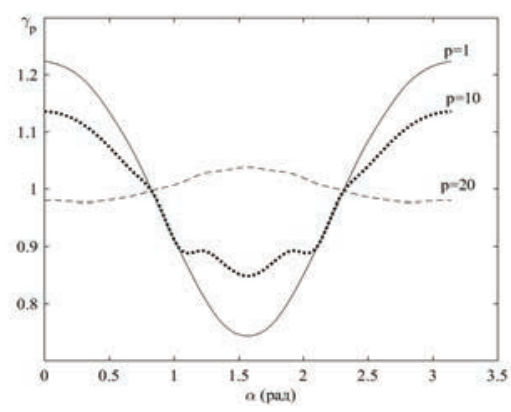

Рис. 1. Нормированный декремент затухания когерентной компоненты мод. 
Угол $\alpha$ между вектором скорости ветра $V$ и направлением акустической трассы рассматривается в диапазоне $0 \leq \alpha \leq \pi$ ввиду очевидной симметрии $\gamma_{\mathrm{p}}(-\alpha) \equiv \gamma_{\mathrm{p}}(\alpha)$. Сразу заметим, что все полученные зависимости в диапазоне $0 \leq \alpha \leq \pi$ также симметричны относительно $\alpha=\pi / 2$. Видно, что зависимость $\gamma_{\mathrm{p}}$ от угла $\alpha$ проявляется максимально для низших слабозатухающих мод волновода (которые и определяют характеристики акустического поля в мелком море). Для мод с высокими номерами $\gamma_{\mathrm{p}}(\alpha)$ практически не зависит от угла $\alpha$. Интересно также отметить, что при $\alpha \approx \pi / 4$ и $\alpha \approx 3 \pi / 4$ упрощенная изотропная модель волнения дает практически правильные результаты. На рис. 2 приведены данные расчетов интенсивности рассеянной компоненты $n_{\mathrm{p}}(\alpha)$ (акустических мод с номерами $p=1,10$ и 20). Здесь также угловая зависимость $n_{\mathrm{p}}(\alpha)$ более выражена для низших мод волновода. При этом отличие результатов расчета для анизотропной модели волнения и упрощенной изотропной более значимо и может достигать 10 дБ.

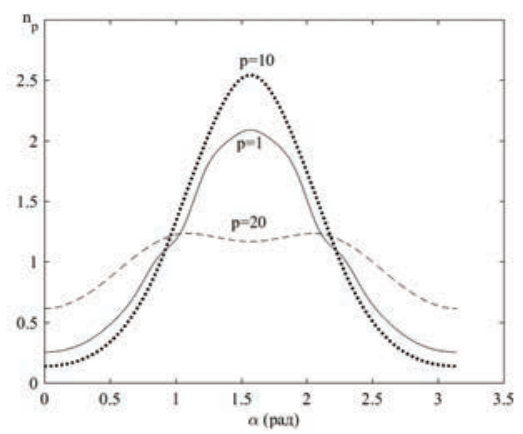

Рис. 2. Нормированная интенсивность рассеянной компоненты мод.

Таким образом, на основании приведенных расчетов можно сделать предварительный вывод, что учет анизотропии ветрового волнения более важен для прогнозирования характеристик рассеянной компоненты акустического поля, чем его когерентная компонента.

Работа выполнена при поддержке гранта РНФ № 20-19-00383.

\section{Литература}

1. Канцельсон Б.Г., Петников В.Г. Акустика мелкого моря. М. Наука. 1997. С. 193.

2. Раевский М.А., Хилько А.И. О пространственно-временной когерентности низкочастотных акустических волн в мелком море с флуктуирующими параметрами // Акуст. журн. 2015. Т. 61. № 3. С. 369-378. 
3. Давидан И.Н., Лопатухин Л.И., Рожков В.А. Ветровое волнение в Мировом океане. Л.: Гидрометеоиздат, 1985. 256 С.

4. Завольский Н.А., Раевский М.А. Горизонтальная анизотропия динамических шумов в глубоком и мелком море // Акуст. журн. 2019. Т. 65. № 2. С. 197-202.

\author{
V.G. Burdukovskaya, M.A. Raevsky
}

\title{
INFLUENCE OF WIND WAVE ANISOTROPY ON THE SCATTERING EFFECTS OF ACOUSTIC MODES IN A SHALLOW SEA
}

\author{
IAPRAS, NizhnyNovgorod, Russia \\ 603950, ul. Ul'yanova, 46, \\ Tel.: (831) 416-0629; E-mail: bvg@appl.sci-nnov.ru
}

\begin{abstract}
A theoretical study of the horizontal anisotropy of the acoustic characteristics of a tonal signal in a waveguide with anisotropic wind waves is carried out. The applicability of the traditional model of isotropic wind waves for applied acoustics is discussed.
\end{abstract}

Keywords: acoustic characteristics, isotropic wind waves

Acknowledgment: This work was supported by the Russian Science Foundation grant No. 20-19-00383.

\section{References}

1. Kantselson B.G. and Petnikov V.G. Shallow Sea Acoustics. Moscow: Nauka, 1997, 193 p.

2. Raevsky M.A. and Khilko A.I. On the space-time coherence of low-frequency acoustic waves in a shallow sea with fluctuating parameters. Akust. Zhurnal, 2015, Vol. 61, No. 3, pp. 369-378.

3. Davidan I.N., Lopatukhin L.I., and Rozhkov V.A. Wind waves in the World Ocean. Leningrad: Hydrometeoizdat, 1985, $256 \mathrm{p}$.

4. Zavolsky N.A. and Raevsky M.A. Horizontal anisotropy of dynamic noise in deep and shallow sea. Akust. Zhurnal, 2019, Vol. 65, No. 2, pp. 197-202. 